OPEN ACCESS

Edited by:

Alan Apter,

Schneider Children's Medical

Center, Israel

Reviewed by:

Amy Krain Roy,

Fordham University, United States

Axel Steiger,

Ludwig Maximilian University of

Munich, Germany

*Correspondence:

Ayelet Armon-Omer

ayelet.o@ziv.health.gov.il

Specialty section:

This article was submitted to

Child and Adolescent Psychiatry,

a section of the journal

Frontiers in Psychiatry

Received: 12 July 2021

Accepted: 13 October 2021

Published: 05 November 2021

Citation

Armon-Omer A, Amir E, Neuman H, Khateeb S, Mizrachi I, Shalan M,

Tamir S and Yatzkar U (2021) Unique Trans-fatty Acid Profile in Children With Attention Deficit Hyperactivity

Disorder

Front. Psychiatry 12:740169. doi: 10.3389/fpsyt.2021.740169

\section{Unique Trans-fatty Acid Profile in Children With Attention Deficit Hyperactivity Disorder}

\author{
Ayelet Armon-Omer ${ }^{1 *}$, Eti Amir ${ }^{2}$, Hadar Neuman ${ }^{3}$, Saleh Khateeb ${ }^{4}$, Itai Mizrachi ${ }^{4}$, \\ Monia Shalan ${ }^{4}$, Snait Tamir ${ }^{5,6}$ and Uri Yatzkar ${ }^{7,8}$ \\ ${ }^{1}$ Research Laboratory, Ziv Medical Center, Zefat, Israel, ${ }^{2}$ Department of Nutrition, Ziv Medical Center, Zefat, Israel, ${ }^{3}$ Medical \\ Laboratory Sciences, Zefat Academic College, Zefat, Israel, ${ }^{4}$ Department of Pediatrics, Ziv Medical Center, Zefat, Israel, \\ ${ }^{5}$ Laboratory of Human Health and Nutrition Sciences, MIGAL Galilee Research Institute, Kiryat Shmona, Israel, ${ }^{6}$ Nutrition \\ Science, Tel-Hai College, Upper Galilee, Israel, ${ }^{7}$ Department of Child and Adolescent Psychiatric, Ziv Medical Center, Zefat, \\ Israel, ${ }^{8}$ Faculty of Medicine, Bar Ilan University, Zefat, Israel
}

Background: Attention deficit hyperactivity disorder (ADHD) is the most common developmental disorder in children. Studies suggest an association between fatty acids composition and ADHD pathogenesis. We aimed to investigate whether children diagnosed with $A D H D$ present unique fatty acid profiles in red blood cells (RBC), as compared to children without ADHD.

Method: We examined 60 children aged 6-14 years, out of which 32 were diagnosed with $\mathrm{ADHD}$, and 28 were not. Blood was collected from all children to quantify an array of 26 fatty acids from RBC membranes. Fatty acid methyl esters were generated by acid transesterification and analyzed by gas chromatography.

Results: We found that children with ADHD presented unique fatty acid profiles on RBC membranes with significantly higher levels of most of the trans-fatty acids (Total trans-fatty acids $0.64 \pm 0.21$ vs. $0.49 \pm 0.18 p=0.003$ ) and lower levels of docosahexaenoic acid (DHA), as compared to controls (4.06 \pm 0.79 vs. $4.68 \pm 1.37 p=0.040$ ). Additionally, total trans-fatty acids were higher in children with extremely severe clinical ADHD condition score, as compared to milder ADHD scores and to control children (0.72 $\pm 0.18,0.64 \pm 0.20,0.61 \pm 0.22,0.49 \pm 0.18, p=0.010$, accordingly).

Conclusion: Children with ADHD have higher levels of trans-fatty acids in RBCs, compared to children without ADHD. This study points to a possible link between trans-fatty acids and ADHD. Understanding these findings and the clinical meaning will potentially contribute to a more targeted dietary intervention.

Keywords: ADHD, trans-fatty acid, CGI-S, red blood cells, DHA

\section{INTRODUCTION}

Attention deficit hyperactivity disorder (ADHD) is the most common developmental disorder in school aged children (1). The prevalence rate of ADHD within Western countries is between 5 and $12 \%$, while about $60 \%$ of these children retain ADHD symptoms into adulthood (2). Attention deficit hyperactivity disorder is defined as a persistent and pervasive pattern of inattention and/or 
hyperactivity-impulsivity that interferes with functioning or development and results in a negative impact on social and academic/occupational activities. Attention deficit hyperactivity disorder is a multifactorial disorder, the cause not precisely known, and its etiology is complex, involving both neurobiological and environmental influence (3). Nutrition is one of the environmental factors possibly involved in ADHD pathogenesis (4). Currently, the exact role of nutrition is unclear, and ADHD therapy is not associated with a particular diet (5). However, ADHD symptoms have been associated and were shown to predict poorer diet quality (6). Exposure to toxins, or food additives such as dyes and preservatives was reported to promote development of ADHD (7).

It has previously been suggested that altered fatty acid composition may be associated with $\operatorname{ADHD}(8,9)$. However, conflicting observations were found regarding fatty acid imbalances in ADHD in the different studies (10). Fatty acids are integral components of biological membrane phospholipids, and influence membrane fluidity, function of receptors, ion transport, and enzyme activity (11). Fatty acids can be saturated or unsaturated (containing double bonds between carbon atoms). Additionally, lower omega-3 polyunsaturated fatty acid (n-3 PUFA) levels were found to positively associate with ADHD symptom severity in children (8). One meta-analysis found that children with ADHD had lower blood levels of reliable omega-3 than typically developing children (12). Therefore, several clinical trials of n-3 PUFAs in children with ADHD have been performed, leading to controversial results. While some clinical trials showed improvement in clinical symptoms (e.g., hyperactivity/impulsivity) and cognitive performances, others did not find beneficial effects (13).

The geometry of the double bonds in lipids biosynthesized by eukaryotes is exclusively cis (14). Trans-fatty acids are often considered to have negative effects on human health $(9,15)$. They are roughly similar to saturated fats in their three-dimensional structure and may influence the melting point and the ability to fit tightly in a membrane structure. Trans-fatty acids could cause inflammation, calcification of arterial cells, and are a wellknown risk factor for cardiovascular diseases (16). A study in rats showed that trans-fatty acids were incorporated into brain structures that were rich in PUFAs, suggesting this can potentially be the case in humans as well (17). Additionally, development of hyperactive behavior in rats was observed after chronic consumption of trans-fatty acids (18). However, we found only one study in humans that suggested that female adolescents with ADHD have a significantly higher intake of trans-fatty acids than those without ADHD (19). Several studies have previously linked fat consumption with ADHD. A study which reported dietary patterns in adolescents with ADHD using complete 7day dietary records demonstrated that ADHD subjects consumed significantly higher levels of trans-fatty acids per day $(3.6 \pm 3.1$ vs. $1.3 \pm 0.7 \mathrm{~g}, p=0.038$ ) (8). Another example is an Australian study which showed that a Western diet, characterized by high intakes of saturated and trans-fat, was associated with ADHD symptoms in children (20).

One of the greatest challenges in research of different central nervous system (CNS) disorders like ADHD is that most CNS tissues are not easily accessible. Therefore, in this study we chose to measure fatty acid profiles from blood. While blood does not fully resemble what occurs within the CNS, it remains the best minimally-invasive source for measuring nutritional components (21). As fatty acids build cell membranes, the fatty acid profile of red blood cells (RBCs) reflects the long term dietary fatty acid intake averaged over the RBC lifespan (up to 120 days) of an individual. The fatty acid profiling method has been previously validated for RBC fatty acid composition. Several alterations, especially regarding levels of omega-3, have been shown in common chronic childhood diseases in Western countries such as asthma, allergy (22), depression (23), and ADHD (24).

Our aims were to test the fatty acid composition of RBC membranes in children with vs. without ADHD, and answer whether children with ADHD present a unique fatty acid profile.

\section{MATERIALS AND METHODS Study Design}

The study was authorized by the Ziv Helsinki Committee, Israel Ministry of Health, and registered at clinicaltrials.gov under number NCT02391428. Written informed consent was obtained from parents, and verbal assent was received from children before entering the study.

\section{Participants}

A total of 60 volunteers between 6 to 14 years of age were recruited through the department of child and adolescent psychiatry at the Ziv Medical Center, Israel. Out of these, 32 children were diagnosed with ADHD as confirmed by a children's psychiatrist according to the criteria of Diagnostic and Statistical Manual of Mental Disorders-5 (DSM-5) (25). Presentation type of $\mathrm{ADHD}$ in the study included: combined presentation ( $n$ $=24,75 \%)$, predominantly inattentive presentation $(n=7$, $21.8 \%)$, and predominantly hyperactive/impulsive presentation $(n=2,6.25 \%)$. In addition, 28 children without ADHD or related neuropsychiatric syndromes were recruited as a control group. Exclusion criteria for both groups included history of other psychiatric disorders and other severe chronic or autoimmune disorders. Body weight and height of subjects were measured by an electronic balanced beam scale and body mass index (BMI) was calculated.

Blood samples $(3 \mathrm{ml})$ were taken from all children by venipuncture into EDTA tubes. RBCs were isolated immediately after collection by centrifugation at $2,500 \mathrm{~g}$ for $10 \mathrm{~min}$. and then stored at $-80^{\circ} \mathrm{C}$ for subsequent analyses. Researchers were blinded for the status of samples when performing analysis.

Fatty acid composition was analyzed by the omega-3 index methodology previously published (26). Blood fatty acid composition was assessed from separated and thawed RBCs by acid transesterification and analyzed by using gas chromatography (GC2010, Shimadzu, Duisburg, Germany) using hydrogen as a carrier gas after extraction and conversion to fatty acid methyl ester. A total of 26 fatty acids were quantified and validated by a standard mixture of fatty acids characteristic of 
RBCs. Results were calculated as a percentage of total identified fatty acids after response factor correction.

\section{ADHD Symptoms Evaluation}

All children were clinically evaluated for ADHD symptoms by psychiatric examination according to the DSM-5 definitions, including the commonly used Clinical Global ImpressionSeverity scale (CGI-S) assessment (27). The CGI-S is a sevenpoint scale that assesses the overall clinical status of a subject, with scores ranging from 1 (mild) to 7 (extremely severe), relative to the clinician's past experience with patients who have the same diagnosis.

\section{Statistical Analysis}

In order to ensure correct use of parametric statistical tests, both the ADHD and the control groups included $\sim 30$ participants as minimum size groups in this pilot study. For categorical variables, summary tables are provided giving sample size and absolute frequencies. For continuous variables, summary tables are provided giving arithmetic mean $(\mathrm{M})$ and standard deviation (SD). The independent-sample $t$-test or Mann-Whitney nonparametric tests were applied for testing differences between control and ADHD groups. Kruskal-Wallis non-parametric tests were used to for testing differences according to CGI-S assessment. $p$-value of $5 \%$ or less was considered statistically significant. All data was analyzed using SPSS version 24 (SPSS Inc., Chicago, IL, USA).

\section{RESULTS}

\section{General Characteristics}

The study included 32 children with ADHD, along with 28 children without $\mathrm{ADHD}$, as a control group. The male-tofemale ratio among ADHD participants was about 2:1 (Table 1), which is in accordance with a higher male ratio in previous epidemiological studies of ADHD (28). Gender at enrollment was comparable between the ADHD vs. control groups (66.7 vs. $50.0 \%$ males, $p=0.187)$. Mean age at enrollment was similar among the ADHD group and the control group $(10.2 \pm 2.0$ vs. $10.3 \pm 2.4$ years, $p=0.876$ ). The mean BMI-value of the
ADHD group was $18.03( \pm 3.5)$, and mean BMI percentile was 57.86 . In the control group, mean BMI-value was $15.68( \pm 1.9)$, and mean BMI percentile was $26.86(p=0.046)$. Both averages and percentiles were within the normal range. Children were diagnosed with ADHD according to the criteria of DSM-5 and the presentation type of ADHD included: combined presentation $(n=23,71.9 \%)$, predominantly inattentive presentation $(n=7$, $21.8 \%)$, and predominantly hyperactive/impulsive presentation $(n=2,6.25 \%)$. There were no significant changes between the different groups.

The children with ADHD either did not receive any medication $(n=19)$, or consumed only methylphenidate medication ( $n=13,40.6 \%$ ), however no significant differences were observed in additional characteristic values between these two sub-groups (Table 2). Importantly, these two groups displayed no differences in CGI-S.

\section{RBC Fatty Acid Profiles}

We assessed whether children with ADHD present unique fatty acid profiles on RBC membranes, as compared to the nonADHD healthy controls. We found significant differences in levels of several fatty acids when comparing the overall ADHD group with controls (Table 3). Out of the 26 measured fatty acids, 11 showed significant differences between groups; children

TABLE 2 | Characteristics according to use of methylphenidate medication.

\begin{tabular}{lccc}
\hline Variables & $\begin{array}{c}\text { No } \\
\text { medication } \\
(\boldsymbol{n}=\mathbf{1 9})\end{array}$ & $\begin{array}{c}\text { Methylphenidate } \\
\text { medication } \\
(\boldsymbol{n}=\mathbf{1 3})\end{array}$ & $\boldsymbol{P}$ \\
\hline Gender, male $(n, \%)$ & $11,57.9$ & $11,84.6$ & 0.141 \\
Age (years, mean $\pm \mathrm{SD})$ & $10.2 \pm 2.3$ & $10.3 \pm 1.8$ & 0.870 \\
Weight $(\mathrm{kg}$, mean $\pm \mathrm{SD})$ & $36.3 \pm 11.5$ & $34.0 \pm 9.5$ & 0.558 \\
Height $(\mathrm{m}$, mean $\pm \mathrm{SD})$ & $1.40 \pm 0.14$ & $1.40 \pm 0.12$ & 0.938 \\
BMI $\left(\mathrm{kg} / \mathrm{m}^{2}\right.$, mean $\left.\pm \mathrm{SD}\right)$ & $18.0 \pm 2.6$ & $17.1 \pm 2.3$ & 0.316 \\
CGl-S $(\mathrm{m}$, mean $\pm \mathrm{SD})$ & $4.8 \pm 0.7$ & $5.1 \pm 0.9$ & 0.448 \\
\hline
\end{tabular}

SD, standard deviation; BMI, body mass index. CGI-S, clinical global impressionseverity scale.

TABLE 1 | Demographic characteristics of the study participants.

\begin{tabular}{|c|c|c|c|c|c|c|c|}
\hline \multirow[t]{2}{*}{ Variables } & \multirow{2}{*}{$\begin{array}{l}\text { Control } \\
(n=28)\end{array}$} & \multirow{2}{*}{$\begin{array}{l}\text { ADHD } \\
(n=32)\end{array}$} & \multirow[t]{2}{*}{$p^{*}$} & \multicolumn{4}{|c|}{ ADHD CGI-S } \\
\hline & & & & $\begin{array}{c}4 \\
(n=10)\end{array}$ & $\begin{array}{c}5 \\
(n=14)\end{array}$ & $\begin{array}{c}6 \\
(n=8)\end{array}$ & $p^{\star \star}$ \\
\hline Gender, male ( $n, \%)$ & $14,50.0$ & $22,68.8$ & 0.187 & $7,70.0$ & $8,57.1$ & $7,87.5$ & 0.349 \\
\hline Methylphenidate medication ( $n, \%)$ & & $13,40.6$ & & $4,40.0$ & $4,28.6$ & $5,62.5$ & 0.385 \\
\hline Age (years, mean $\pm S D$ ) & $10.3 \pm 2.4$ & $10.2 \pm 2.0$ & 0.876 & $11.1 \pm 1.8$ & $9.9 \pm 2.2$ & $9.8 \pm 2.0$ & 0.233 \\
\hline Weight (Kg, mean \pm SD) & $31.6 \pm 10.9$ & $36.1 \pm 11.5$ & 0.156 & $37.9 \pm 10.4$ & $36.5 \pm 11.6$ & $30.1 \pm 8.4$ & 0.245 \\
\hline Height (m, mean $\pm \mathrm{SD})$ & $1.36 \pm 0.17$ & $1.40 \pm 0.13$ & 0.429 & $1.44 \pm 0.09$ & $1.40 \pm 0.15$ & $1.34 \pm 0.12$ & 0.303 \\
\hline $\mathrm{BMl}\left(\mathrm{Kg} / \mathrm{m}^{2}\right.$, mean $\left.\pm \mathrm{SD}\right)$ & $15.67 \pm 1.92$ & $18.03 \pm 3.45$ & 0.046 & $18.1 \pm 3.4$ & $18.0 \pm 2.0$ & $16.4 \pm 1.6$ & 0.186 \\
\hline
\end{tabular}

$A D H D$, attention-deficit hyperactivity disorder; CGI-S, clinical global impression-severity scale; SD, standard deviation; BMI, body mass index.

*The independent-sample t-test.

${ }^{\star *}$ Kruskal-Wallis non-parametric tests were used. 
TABLE 3 | Fatty acid profiles from RBC membranes comparing children with ADHD vs. without (control).

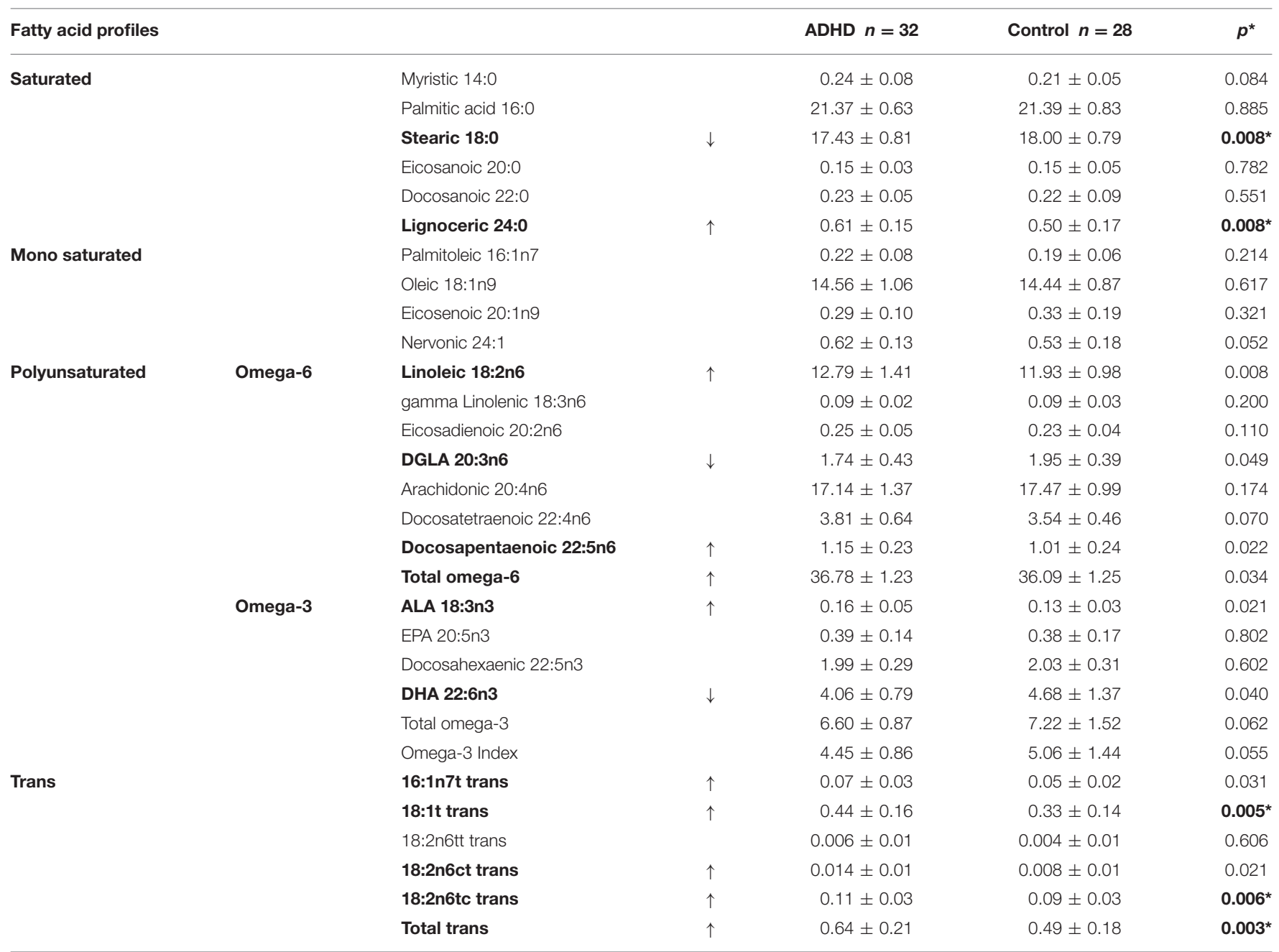

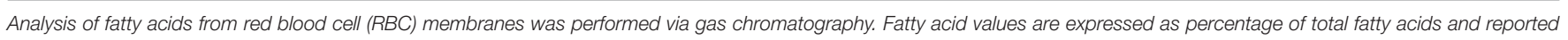

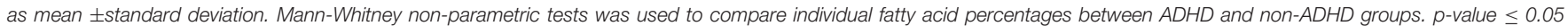

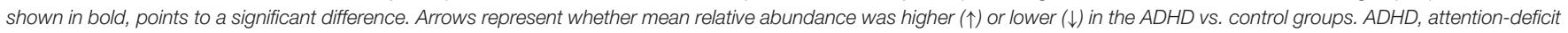

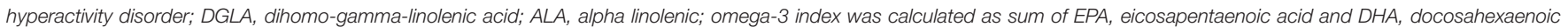

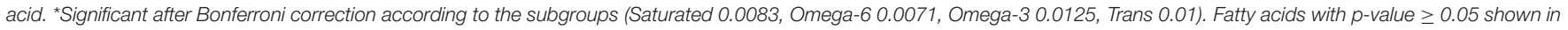
bold.

with ADHD presented lower levels of three fatty acids, and higher levels of eight fatty acids, as compared to controls. After Bonferroni correction according to the subgroups, significance was remained only in three trans-fatty acids $(0.05 / 5=0.01)$ and two saturated fatty acids $(0.05 / 6=0.0083)$ significant indices, as can be seen in Table 3. Percentages of the saturated fat lignoceric acid (24:0), and most types of trans-fatty acids (18:1t, 18:2n6ct, and $18: 2 \mathrm{n} 6 \mathrm{tc}$ ) were significantly higher in children with vs. without ADHD (Table 3). The monounsaturated fat nervonic acid (24:1n9), showed a trend toward significance with higher levels in ADHD vs. control groups. The levels of Stearic acid (18:0) were significantly lower in the ADHD group, compared to children without ADHD (Table 3). The average omega-3 index of children diagnosed with ADHD was slightly lower (4.45\%) compared to children without ADHD (5.06\%). This difference was close to significance ( $p=0.055$, Table 3 ). The levels of total trans-fatty acids within the ADHD group did not differ between children receiving medication and those that do not $(0.71 \pm 0.18$ vs. $0.61 \pm 0.20, p=0.126)$.

We next analyzed the levels of trans-fatty acids according to CGI-S scale assessment by psychiatric examination. We found that the percentage of total trans-fats was higher in the extremely severe clinical condition score CGI-S $=6$, and CGI-S $=5$, as compared to control subjects.

\section{DISCUSSION}

Our study aimed to evaluate the association between the RBC fatty acid profile and ADHD symptomatology, as measured in a sample of Israeli children with vs. without ADHD.

We identified various alterations in the fatty acid profile of children with ADHD compared to controls. Three types of transfatty acids were represented by significantly higher levels in ADHD vs. control children. Additionally, the percentage of total 
trans-fats was higher in the extremely severe ADHD, and in milder ADHD as compared to control subjects.

Concerning the composition of saturated fatty acids, we found higher levels of lignoceric acid (24:0), whereas significantly lower levels of stearic acid (18:0) in children with ADHD. Interestingly, high levels of lignoceric acid in RBCs were previously found to be associated with inflammation in adults with metabolic syndrome (29). Decreased levels of the serum sphingomyelin 18:0 were previously found in ADHD patients (30). Regarding monounsaturated fatty acids, nervonic acid (24:1n9) showed a trend toward significance with higher levels in the ADHD group vs. controls. These findings are in contrast to previous work showing lower levels of nervonic acid in RBC membranes in children with vs. without ADHD in Taiwan (31). Interestingly, according to the literature, nervonic acid was previously suggested as a good candidate biomarker for a depressive state (30), as well as a predictor for conversion to psychosis in young people at high clinical risk for psychosis (31). However, the clinical relevance of nervonic acid to ADHD remains unknown.

The mean omega-3 percentage in healthy Israeli children was found in our study to be $5.06 \%$, which is significantly higher than values reported for healthy children from some other countries $(3.5 \%$ in children in the United States, and $4.9 \%$ in Australian adolescents), while lower than the index of $5.7 \%$ found in adolescents in Norway (32). This may be important to take into account when comparing values from studies performed on different populations around the globe, and may reflect the different diets of children at various geographical locations.

While not reaching significance, we found a lower mean omega-3 index in children with ADHD (4.45\%), compared to the non-ADHD control group. We additionally found that levels of the docosahexaenoic acid (DHA) component of the omega3 index, the main omega- 3 in the CNS, were lower in the ADHD group, compared to the control group without ADHD. However, Eicosapentaenoic acid levels did not differ significantly between groups.

In relation to the omega- 6 family, levels of linoleic acid and docosapentaenoic acid were higher in the ADHD group vs. controls. The only omega- 6 that was lower in children with ADHD vs. controls was dihomo-gamma-linolenic acid (DGLA), matching results of a previous study among hyperactive children (33). Additionally, lower DHA and DGLA levels, as well as higher linoleic acid levels, were previously found in ADHD adolescents when compared to age-matched control subjects (9). Dihomo-gamma-linolenic acid is an omega-6, which can be further converted into anti-inflammatory prostaglandin. It has been suggested that DHA and DGLA and their metabolites have pro-immune actions in addition to their prominent role in the brain (34).

Although values of BMI were within the normal range in both study groups, it is important to note that a significant difference in BMI was observed in children with vs. without ADHD, with a higher average within the ADHD group. This result has also been found by others (35), suggested that the etiological factors influencing the association between ADHD and BMI may differ by demographic characteristics (36). Mechanistically, there may be a connection between the higher trans-fatty acid levels we found in the ADHD group and the higher BMI levels, but no significant correlations found here. It has been shown in monkeys that long-term trans-fatty acid consumption is an independent factor in weight gain (37). A rat study which aimed to test the effects of trans-fatty acids on markers of obesity and pre-diabetic state suggested that trans-fatty acids altered nutrient handling in liver, adipose tissue, and skeletal muscle as well as the mechanism by which trans-fatty acids induce insulin resistance (38). There have also been some epidemiological studies showing the same trends (39). However, the precise potential mechanisms linking between ADHD, trans-fatty acids, and higher BMI require further investigation (40). For instance, it is of particular interest whether the dietary consumption levels of trans-fatty acids differ between children with vs. without ADHD.

In this study, we chose measurements of blood trans-fatty acid levels, as these are generally more reliable and comprehensive than self-reported intake assessment, which are limited in the ability to accurately reflect the nutrient consumption of individuals due to measurement error, recall bias, selective report, and incompleteness of food composition databases (41). If indeed the relatively high levels of trans-fatty acids observed in RBCs also reflect high levels in brain cell membranes, they may perhaps take part in the pathophysiology of ADHD. The physiological implication could be due to trans-fatty acids interfering with the desaturation and elongation of both omega- 6 and omega3 fatty acids, thus further decreasing their availability for metabolism. Another possibility is that since trans-molecules are more static, they may reduce the flexibility of the membranes. The mechanism by which trans-fatty acid isomers influence health outcomes remains elusive (42). One suggestion is that the toxicity of trans-fatty acids may reside in their ability to inhibit cytoprotective stress responses induced by saturated fatty acids (43). Our study is unique as only few studies on trans-fatty acids in children have been performed previously. Despite the correlation found between fatty acid profiles and ADHD status, it remains unknown whether increased trans-fatty acid levels can cause or exacerbate ADHD, or whether higher trans-fatty acid levels in blood are a result of a contributory mechanism. Higher levels of trans-fatty acids may be a result either of higher dietary consumption or altered metabolism of fatty acids.

In general, our results present a unique RBC fatty acid profile for children with vs. without ADHD, highlighting observed differences in specific fatty acids. Due to the numerous differences, it remains difficult to assess the precise clinical effect of each fatty acid separately by this analysis. Additionally, our study is observational, therefore it cannot provide causal evidence of an effect of trans-fatty acids on the development of health outcomes examined; it can only describe associations. However, certain observations, such as the relatively high levels of trans-fatty acids in children with ADHD present potential directions for future research and potential interventions.

\section{CONCLUSIONS}

Our study reveals significant differences in the composition of RBC fatty acids in children with vs. without ADHD. 
Higher relative levels of most trans-fatty acids were found in children with ADHD vs. control. Future studies should be designed to evaluate the clinical relevance of trans-fatty acids in a larger cohort of ADHD subjects, together with dietary analysis, and perhaps evaluate elimination of trans-fats as a nutritional intervention.

\section{DATA AVAILABILITY STATEMENT}

The raw data supporting the conclusions of this article will be made available by the authors, without undue reservation.

\section{ETHICS STATEMENT}

The studies involving human participants were reviewed and approved by Ziv Helsinki Committee, Israel Ministry of Health. Written informed consent to participate in this study was provided by the participants' legal guardian/next of kin.

\section{REFERENCES}

1. Singh A, Yeh CJ, Verma N, Das AK. Overview of attention deficit hyperactivity disorder in young children. Health Psychol. Res. (2015) 3:2115. doi: 10.4081/hpr.2015.2115

2. Davidovitch M, Koren G, Fund N, Shrem M, Porath A. Challenges in defining the rates of ADHD diagnosis and treatment: trends over the last decade. $B M C$ Pediatr. (2017) 17:218. doi: 10.1186/s12887-017-0971-0

3. Rappley MD. Clinical practice. Attention deficit-hyperactivity disorder. $N$ Engl J Med. (2005) 352:165-73. doi: 10.1056/NEJMcp032387

4. Curtis LT, Patel K. Nutritional and environmental approaches to preventing and treating autism and attention deficit hyperactivity disorder (ADHD): a review. J Altern Complement Med. (2008) 14:79-85. doi: 10.1089/acm.2007.0610

5. Sarris J, Kean J, Schweitzer I, Lake J. Complementary medicines (herbal and nutritional products) in the treatment of Attention Deficit Hyperactivity Disorder (ADHD): a systematic review of the evidence. Complement Ther Med. (2011) 19:216-27. doi: 10.1016/j.ctim.2011.06.007

6. Mian A, Jansen PW, Nguyen AN, Bowling A, Renders CM, Voortman T. Children's attention-deficit/hyperactivity disorder symptoms predict lower diet quality but not vice versa: results from bidirectional analyses in a population-based cohort. J Nutr. (2019) 149:642-8. doi: 10.1093/jn/nxy273

7. Braun JM, Kahn RS, Froehlich T, Auinger P. Lanphear BP. Exposures to environmental toxicants and attention deficit hyperactivity disorder in US children. Environ Health Perspect. (2006) 114:1904-9. doi: 10.1289/ehp.9478

8. Colter AL, Cutler C, Meckling KA. Fatty acid status and behavioural symptoms of attention deficit hyperactivity disorder in adolescents: a casecontrol study. Nutr J. (2008) 7:8. doi: 10.1186/1475-2891-7-8

9. Ghezzo A, Visconti P, Abruzzo PM, Bolotta A, Ferreri C, Gobbi $G$, et al. Oxidative stress and erythrocyte membrane alterations in children with autism: correlation with clinical features. PLoS ONE. (2013) 8:e66418. doi: 10.1371/journal.pone.0066418

10. LaChance L, McKenzie K, Taylor VH, Vigod SN. Omega-6 to omega-3 fatty acid ratio in patients with ADHD: a meta-analysis. J Can Acad Child Adolesc Psychiatry. (2016) 25:87-96.

11. Ernst R, Ballweg S, Levental I. Cellular mechanisms of physicochemical membrane homeostasis. Curr Opin Cell Biol. (2018) 53:4451. doi: 10.1016/j.ceb.2018.04.013

12. Hawkey E, Nigg JT. Omega-3 fatty acid and ADHD: blood level analysis and meta-analytic extension of supplementation trials. Clin Psychol Rev. (2014) 34:496-505. doi: 10.1016/j.cpr.2014.05.005

13. Chang JP, Su KP. Nutritional neuroscience as mainstream of psychiatry: the evidence- based treatment guidelines for using omega-3 fatty acids as
Written informed consent was obtained from the minor(s)' legal guardian/next of kin for the publication of any potentially identifiable images or data included in this article.

\section{AUTHOR CONTRIBUTIONS}

AA-O, EA, ST, and UY: designed research. AA-O, EA, KS, IM, MS, and UY: conducted research. AA-O and $\mathrm{HN}$ : analyzed data and wrote the paper. All authors read and approved the final manuscript.

\section{ACKNOWLEDGMENTS}

The authors would like to thank to Prof. Clemens von Schacky (University of Munich, Germany) for his HS-Omega-3 index analysis. The authors would like to thank Ms. Adi Sharabi-Nov for her statistical work. Finally, the authors would like to thank all participating children and parents in this study. a new treatment for psychiatric disorders in children and adolescents. Clin Psychopharmacol Neurosci. (2020) 18:469-83. doi: 10.9758/cpn.2020.18.4.469

14. Chatgilialoglu C, Ferreri C, Melchiorre M, Sansone A, Torreggiani A. Lipid geometrical isomerism: from chemistry to biology and diagnostics. Chem Rev. (2014) 114:255-84. doi: 10.1021/cr4002287

15. Li H, Zhang Q, Song J, Wang A, Zou Y, Ding L, et al. Plasma trans-fatty acids levels and mortality: a cohort study based on 1999-2000 National Health and Nutrition Examination Survey (NHANES). Lipids Health Dis. (2017) 16:176. doi: 10.1186/s12944-017-0567-6

16. Wanders AJ, Zock PL, Brouwer IA. Trans fat intake and its dietary sources in general populations worldwide: a systematic review. Nutrients. (2017) 9:840. doi: $10.3390 /$ nu9080840

17. Grandgirard A, Bourre JM, Julliard F, Homayoun P, Dumont O, Piciotti M, et al. Incorporation of trans long-chain n-3 polyunsaturated fatty acids in rat brain structures and retina. Lipids. (1994) 29:251-8. doi: 10.1007/BF025 36329

18. Pase CS, Roversi K, Trevizol F, Kuhn FT, Dias VT, Vey LT, et al. Chronic consumption of trans fat can facilitate the development of hyperactive behavior in rats. Physiol. Behav. (2015) 139:344-50. doi: 10.1016/j.physbeh.2014.11.059

19. Kim JH, Nam CM, Kim JW, Lee DC, Shim JS, Lee HR. Relationship between attention-deficit/hyperactivity disorder and trans fatty acids intake in female adolescents. Acta Paediatr. (2012) 101:e431-3. doi: 10.1111/j.1651-2227.2012.02726.x

20. Howard AL, Robinson M, Smith GJ, Ambrosini GL, Piek JP, Oddy WH, et al. is associated with a "Western" dietary pattern in adolescents. J Atten Disord. (2011) 15:403-11. doi: 10.1177/1087054710365990

21. Zlokovic BV. The blood-brain barrier in health and chronic neurodegenerative disorders. Neuron. (2008) 57:178201. doi: 10.1016/j.neuron.2008.01.003

22. Wilczynska-Kwiatek A, Bargiel-Matusiewicz K, Lapinski L. Asthma, allergy, mood disorders, and nutrition. Eur J Med Res. (2009) 14(Suppl 4):24854. doi: 10.1186/2047-783X-14-S4-248

23. Hibbeln JR. Fish consumption and major depression. Lancet. (1998) 351:1213. doi: 10.1016/S0140-6736(05)79168-6

24. Derbyshire E. Do omega-3/6 fatty acids have a therapeutic role in children and young people with ADHD? J Lipids. (2017) 2017:6285218. doi: 10.1155/2017/6285218

25. Diagnostic and Statistical Manual of Mental Disorders Fifth Edition DSM-5TM. Washington, DC; London: American Psychiatric Association (2013).

26. Harris WS, Von Schacky C. The omega-3 index: a new risk factor for death from coronary heart disease? Prev Med. (2004) 39:21220. doi: 10.1016/j.ypmed.2004.02.030 
27. Busner J, Targum SD. The clinical global impressions scale: applying a research tool in clinical practice. Psychiatry (Edgmont). (2007) 4:28-37.

28. Ramtekkar UP, Reiersen AM, Todorov AA, Todd RD. Sex and age differences in attention-deficit/hyperactivity disorder symptoms and diagnoses: implications for DSM-V and ICD-11. J Amer Acad Child Adolesc Psychiatry. (2010). 49:217.e1-3-28.e1-3. doi: 10.1016/j.jaac.2009.11.011

29. Matsumori R, Miyazaki T, Shimada K, Kume A, Kitamura Y, Oshida K, et al. High levels of very long-chain saturated fatty acid in erythrocytes correlates with atherogenic lipoprotein profiles in subjects with metabolic syndrome. Diabetes Res Clin Pract. (2013). 99:12-8. doi: 10.1016/j.diabres.2012. 10.025

30. Henriquez-Henriquez MP, Solari S, Quiroga T, Kim BI, Deckelbaum RJ, Worgall TS. Low serum sphingolipids in children with attention deficit-hyperactivity disorder. Front Neurosci. (2015) 9:300. doi: 10.3389/fnins.2015.00300

31. Chen JR, Hsu SF, Hsu CD, Hwang LH, Yang SC. Dietary patterns and blood fatty acid composition in children with attentiondeficit hyperactivity disorder in Taiwan. J Nutr Biochem. (2004) 15:467-72. doi: 10.1016/j.jnutbio.2004.01.008

32. Handeland K, Skotheim S, Baste V, Graff IE, Froyland L, Lie O, et al. The effects of fatty fish intake on adolescents' nutritional status and associations with attention performance: results from the FINS-TEENS randomized controlled trial. Nutr J. (2018) 17:30. doi: 10.1186/s12937-0180328-z

33. Mitchell EA, Aman MG, Turbott SH, Manku M. Clinical characteristics and serum essential fatty acid levels in hyperactive children. Clin Pediatr (Phila). (1987) 26:406-11. doi: 10.1177/000992288702600805

34. Das UN. Arachidonic acid and other unsaturated fatty acids and some of their metabolites function as endogenous antimicrobial molecules: a review. J $A d v$ Res. (2018) 11:57-66. doi: 10.1016/j.jare.2018.01.001

35. Khalife N, Kantomaa M, Glover V, Tammelin T, Laitinen J, Ebeling $\mathrm{H}$, et al. Childhood attention-deficit/hyperactivity disorder symptoms are risk factors for obesity and physical inactivity in adolescence. $J$ Am Acad Child Adolesc Psychiatry. (2014) 53:425-36. doi: 10.1016/j.jaac.2014. 01.009

36. Do EK, Haberstick BC, Williams RB, Lessem JM, Smolen A, Siegler IC, et al. The role of genetic and environmental influences on the association between childhood ADHD symptoms and BMI. Int J Obes (Lond). (2019) 43:33-42. doi: 10.1038/s41366-018-0236-5
37. Kavanagh K, Jones KL, Sawyer J, Kelley K, Carr JJ, Wagner JD, et al. Trans fat diet induces abdominal obesity and changes in insulin sensitivity in monkeys. Obesity (Silver Spring). (2007) 15:1675-84. doi: 10.1038/oby.2007.200

38. Dorfman SE, Laurent D, Gounarides JS Li X, Mullarkey TL, Rocheford EC, et al. Metabolic implications of dietary trans-fatty acids. Obesity (Silver Spring). (2009) 17:1200-7. doi: 10.1038/oby.2008.662

39. Thompson AK, Minihane AM, Williams CM. Trans fatty acids and weight gain. Int J Obes (Lond). (2011) 35:315-24. doi: 10.1038/ijo. 2010.141

40. Cortese S. The Association between ADHD and obesity: intriguing, progressively more investigated, but still puzzling. Brain Sci. (2019). 9:256. doi: 10.3390/brainsci9100256

41. Bingham SA, Luben R, Welch A, Wareham N, Khaw KT, Day N. Are imprecise methods obscuring a relation between fat and breast cancer? Lancet. (2003) 362:212-4. doi: 10.1016/S0140-6736(03)13913-X

42. Oteng AB, Kersten S. Mechanisms of action of trans fatty acids. Adv Nutr. (2020) 11:697-708. doi: 10.1093/advances/nmz125

43. Sauvat A, Chen G, Muller K, Tong M, Aprahamian F, Durand S, et al. Transfats inhibit autophagy induced by saturated fatty acids. EBioMed. (2018) 30:261-72. doi: 10.1016/j.ebiom.2018.03.028

Conflict of Interest: The authors declare that the research was conducted in the absence of any commercial or financial relationships that could be construed as a potential conflict of interest.

Publisher's Note: All claims expressed in this article are solely those of the authors and do not necessarily represent those of their affiliated organizations, or those of the publisher, the editors and the reviewers. Any product that may be evaluated in this article, or claim that may be made by its manufacturer, is not guaranteed or endorsed by the publisher.

Copyright (c) 2021 Armon-Omer, Amir, Neuman, Khateeb, Mizrachi, Shalan, Tamir and Yatzkar. This is an open-access article distributed under the terms of the Creative Commons Attribution License (CC BY). The use, distribution or reproduction in other forums is permitted, provided the original author(s) and the copyright owner(s) are credited and that the original publication in this journal is cited, in accordance with accepted academic practice. No use, distribution or reproduction is permitted which does not comply with these terms. 